\title{
Assessment of the Clinical Interpreter of Death in Life-Threatening Infective Cases Admitted in the Intensive Care Unit of a North-Eastern State of India
}

Pollov Borah ${ }^{1}$, Dilip K. Saloi ${ }^{1}$, Amarendra Deka ${ }^{2}$, Rajib Hazarika Sr. ${ }^{1}$, Ranjumoni Konwar ${ }^{3}$, Putul Mahanta Sr. ${ }^{4}$, Deepjyoti Kalita ${ }^{5}$, Chiranjita Phukan Sr. ${ }^{6}$, Kahua Das ${ }^{7}$

\begin{abstract}
1. Anaesthesiology, Jorhat Medical College and Hospital, Jorhat, IND 2. Anaesthesiology, Assam Medical College and Hospital, Dibrugarh, IND 3. Radiology, Fakhruddin Ali Ahmed Medical College (FAAMC) and Hospital, Barpeta, IND 4. Forensic Medicine and Toxicology, Assam Medical College and Hospital, Dibrugarh, IND 5. Microbiology, All India Institute of Medical Sciences, Rishikesh, IND 6. Internal Medicine, Tezpur Medical College and Hospital, Tezpur, IND 7. Physiology, Tezpur Medical College and Hospital, Tezpur, IND
\end{abstract}

Corresponding author: Putul Mahanta Sr., drpmahanta@gmail.com

\section{Abstract \\ Objectives}

The clinical factors affecting a patient's condition monitored over time could be useful not only to decide on an intervention that may increase the patients' possibilities of survival but also to predict the treatment outcome. Therefore, this study evaluates the clinical factors as predictors of mortality among severe sepsis patients admitted in the intensive care unit (ICU) of a tertiary care center.

\section{Method}

We did a prospective study on over 50 life-threatening infective cases with different causes admitted in the ICU. Clinical and biochemical parameters like temperature, heart rate, blood pressure, bicarbonate levels, blood lactate levels, and pH were monitored at admission, after 24 hours, and after 72 hours. The statistical analysis was done using Microsoft Excel (Microsoft Corporation, Redmond, WA) and the Statistical Package for the Social Studies (SPSS) version 22 (IBM Corp., Armonk, NY). We have obtained ethical clearance from the ethics committee (human) of Assam Medical College and Hospital, Dibrugarh. Before the collection of the data, we also took informed consent from the participants.

\section{Results}

The mean age of non-survivors was $44.35 \pm 11.64$ years and that of survivors was $36.60 \pm 9.28$ years, and the difference was statistically significant ( $\mathrm{p}$-value $<0.003$ ). An analysis of values of the various vital signs indicated substantial differences in the mean at different time intervals among survivors and non-survivors (p-value $<0.05$ ). Among non-survivors, mean temperature, pulse, and rate of respiration were observed to increase over time while blood pressure and oxygen saturation levels were significantly decreasing.

Review began 02/24/2021 Review ended 03/13/2021 Published 03/16/2021

\section{(c) Copyright 2021}

Borah et al. This is an open access article distributed under the terms of the Creative Commons Attribution License CC-BY 4.0., which permits unrestricted use, distribution, and reproduction in any medium, provided the original author and source are credited.
Compared to survivors, the mean lactate levels of non-survivors at different time intervals were statistically significant ( $\mathrm{p}$-value $<0.05$ ). It is also observed that the $\mathrm{pH}$ of non-survivors was lower than survivors, and the mean $\mathrm{pH}$ value significantly different at different time intervals among the two groups ( $\mathrm{p}$-value $<0.05$ ).

\section{Conclusion}

The temperature, pulse, rate of respiration, blood pressure, and oxygen saturation levels are essential determinants of patient mortality in those suffering from a severe infection, besides serial lactate levels, bicarbonate levels, and pH levels.

Categories: Anesthesiology, Emergency Medicine, Infectious Disease

Keywords: sepsis, mortality, critical care centre, vital signs, severe sepsis

\section{Introduction}

Life-threatening infective conditions are the foremost causes of morbidity and mortality globally, comprising $20 \%$ to $30 \%$ of deaths in the current clinical practice [1-2]. Infection in patients resulting in systemic inflammatory response syndrome (SIRS) [3] is associated with a rise in body temperature and heart and respiratory rate. It decreases the partial arterial pressure of carbon dioxide $<32 \mathrm{mmHg}$, which predicts the fate of the individual's infective status. Also, the condition of severe sepsis is allied with organ dysfunction. So, in septic shock, vasopressors need to maintain a mean arterial pressure of $>65 \mathrm{mmHg}$, and the serum lactate level is required to be $<2 \mathrm{mmol} / \mathrm{l}[4]$. 
Therefore, these variables in censoriously ill patients predict the severity of the illness and death and evaluate the treatment costs. They also prescribe further required medical intervention besides screening the suitability and effectiveness of the ongoing course of remedial measures. A single clinical variable is unlikely to predict morbidity and mortality outcomes, hence the need for a group of clinical outcomes may come the closest in predicting morbidity and mortality for the patient suffering from sepsis [5].

The persistent rise of blood lactate level in the septicemic patient undergoing treatment in a critical care center (CCC) signifies hypoxic hypoxia and stagnant hypoxia and predicts death [5-7]. The delay of its clearance is observed with increased mortality. So, it is useful as a predictor of fatality and termination of therapy [8].

The current knowledge base for predicting morbidity and mortality following sepsis is still inadequate to implicate in this underdeveloped region of India. The conducted research, even if it exemplifies an essential aid for our knowledge of the issue of patients with severe sepsis, does not resolve the difficulties faced by us in determining the low-cost treatment while considering the affordability of saving the patient's life. Therefore, we evaluated the patients' clinical predictors, along with blood lactate levels, following septicemia and admission to the CCC.

\section{Materials And Methods}

A prospective study was carried out in the critical care center of the anesthesiology department. The study participants $(n=50)$ were all major ( $>18$ years), were suffering from sepsis, and have followed the natural course. Out of the cases studied, 20 suffered from respiratory failure, 15 from cardiovascular collapse, 10 from chronic renal failure, and five cases belonged to other categories. The heart rates, respiratory rate, urine output, and mean blood pressure were recorded hourly in the CCC during the course of treatment.

Vital parameters like oxygen saturation, partial pressure of oxygen (PO2), partial pressure of $\mathrm{CO} 2$ (pCO2), proton concentration $(\mathrm{pH})$, bicarbonate ion ( $\mathrm{HCO} 3$ ), and blood lactate level on admission, at 24 hours, and at 72 hours was monitored and recorded. Blood lactate level was analyzed by using the arterial blood gas $(A B G)$ analyzer and was recorded. Each patient was monitored for four weeks to be registered as a survivor or non-survivor. The present study has followed the Surviving Sepsis Campaign: International Guidelines for Management of Sepsis and Septic Shock: 2018 [9] guidelines to correct the sepsis markers described in the current study.

The patients were physically examined, and the data collected for statistical analysis, which was performed using the Statistical Package for the Social Sciences (SPSS) software version 22 (IBM Corp., Armonk, NY). Descriptive statistical methods were computed, and the student's t-test tested statistical significance. A pvalue of less than 0.05 was considered statistically significant. We have obtained ethical clearance from the ethics committee (human) of Assam Medical College and Hospital (NO. AMC/EC/PG/100). Before collecting the data, we also took informed consent from the participants who could understand and sign the consenting form. In other cases, informed consent was obtained from the legal guardians.

\section{Results}

Out of 50 patients, 27 died during the four-week follow-up treatment, and $20 \%$ of the patients belonged to the age group of 45-50 years, as shown in Figure 1. The mean age of patients was $45.06 \pm 3.26$ years. The mean $( \pm)$ age of non-survivors was $44.35( \pm 11.64)$ years, and in survivors, it was $36.60( \pm 9.28)$ years. This result demonstrates the association of a higher age group with bad outcomes. The differences in mean age in the survivors and non-survivors groups were found to be statistically significant ( $p$-value $<0.003$ ). 


\section{Cureus}

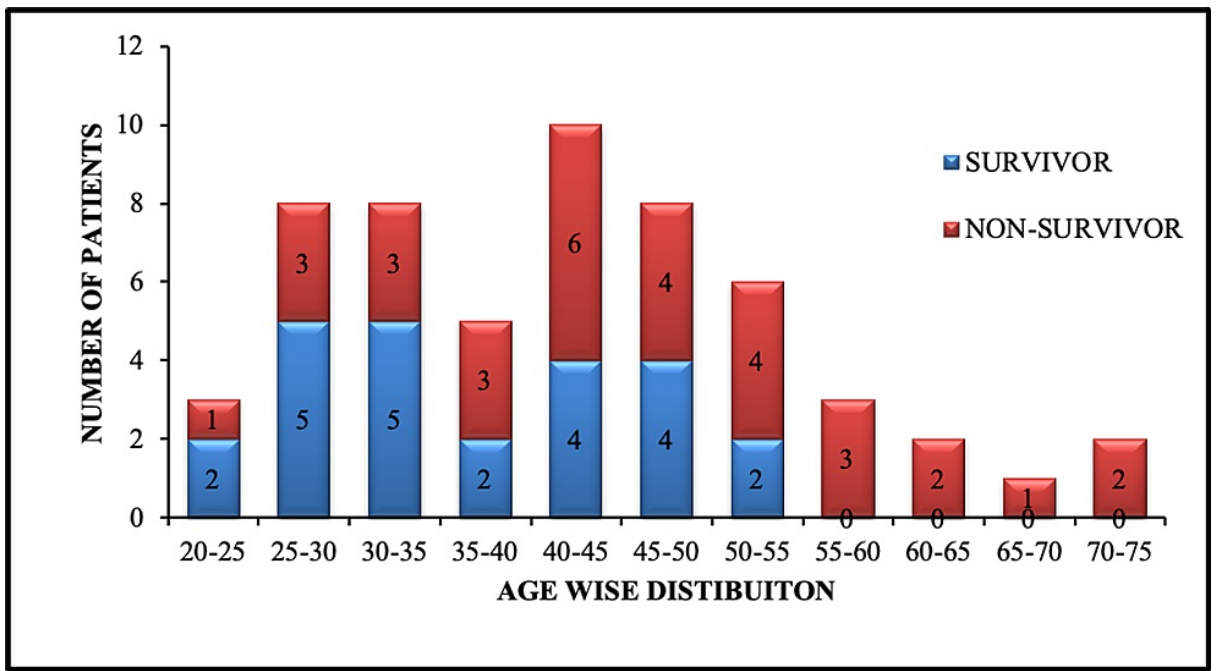

FIGURE 1: Age distribution among survivors and non-survivors

\section{Changes in vital signs}

Changes in vital signs monitored over time among the survivors and non-survivors groups are shown in Table 1. Significant differences in mean were observed in temperature, heart rate, rate of respiration, oxygen saturation levels between the two groups at the time of admission ( $p$-value $<0.05$ ). Patients' mean body temperature was significantly different between the survivor and non-survivor groups at 24 hours and 72 hours with a gradual increase in temperature among non-survivors $(100.530 \pm 2.5301)$ as compared to survivors (98.778 \pm 1.0505$)$. A low level of mean blood pressure was observed among non-survivors as compared to survivors, and the difference was statistically significant at 24 hours and 72 hours. The mean blood pressure decreased from $66.07 \pm 7.820$ to $50.78 \pm 8.679$ in non-survivors. An increasing trend was observed in mean pulse and rate of respiration among non-survivors with increasing time. Those were significantly different from those of survivors at different time intervals ( $\mathrm{p}$-value < 0.05). Oxygen saturation was substantially lower among non-survivors with a mean SpO2 level of $92.19 \pm 9.540$ at admission, $92.19 \pm 10.141$ at 24 hours, and $91.70 \pm 10.622$ at 72 hours while the mean SpO2 levels among survivors were $>$ 95 at various time intervals. A significant decrease in urine output was also observed among non-survivors. The mean urine output among survivors and non-survivors was significantly different at 24 hours and 72 hours (p-value $<0.05)$.

\begin{tabular}{|c|c|c|c|c|c|c|c|c|c|}
\hline \multirow{2}{*}{ Vitals } & \multicolumn{3}{|l|}{ At admission } & \multicolumn{3}{|l|}{24 Hours } & \multicolumn{3}{|l|}{72 Hours } \\
\hline & Survivor & Non- survivor & p-value & Survivor & Non- survivor & $p$-value & Survivor & Non- survivor & $p$-value \\
\hline Temperature & $97.743 \pm 1.1920$ & $98.793 \pm 1.2029$ & 0.003 & $98.535 \pm 1.0998$ & $99.778 \pm 1.8727$ & 0.007 & $98.778 \pm 1.0505$ & $100.530 \pm 2.5301$ & $<0.001$ \\
\hline Mean Arterial Pressure & $71.52 \pm 7.501$ & $66.07 \pm 7.820$ & 0.16 & $73.70 \pm 5.764$ & $57.15 \pm 6.938$ & $<0.001$ & $73.57 \pm 7.668$ & $50.78 \pm 8.679$ & $<0.001$ \\
\hline Heart Rate & $86.48 \pm 9.638$ & $95.74 \pm 9.330$ & 0.01 & $88.70 \pm 13.492$ & $116.3 \pm 12.375$ & $<0.001$ & $89.30 \pm 14.778$ & $130.59 \pm 16.230$ & $<0.001$ \\
\hline Respiratory Rate & $22.52 \pm 1.344$ & $24.19 \pm 1.688$ & $<0.001$ & $22.78 \pm 1.858$ & $26.41 \pm 2.664$ & $<0.001$ & $23.22 \pm 2.504$ & $29.26 \pm 3.748$ & $<0.001$ \\
\hline Oxygen Saturation $\left(\mathrm{SpO}_{2}\right)$ & $96.87 \pm 2.341$ & $92.19 \pm 9.540$ & 0.026 & $97.83 \pm 1.435$ & $92.19 \pm 10.141$ & 0.011 & $98.17 \pm 1.466$ & $91.70 \pm 10.622$ & 0.006 \\
\hline Urine Output & $41.78 \pm 14.666$ & $44.00 \pm 59.283$ & 0.86 & $50.30 \pm 17.867$ & $27.59 \pm 10.903$ & $<0.001$ & $46.91 \pm 16.684$ & $21.52 \pm 12.274$ & $<0.001$ \\
\hline
\end{tabular}

TABLE 1: Vital signs of survivors and non-survivors over time

\section{Lactate value among study participants}

Figure 2 represents the distribution of study participants with different lactate values. The lactate value in survivors ranged from 0.43 to $5.69 \mathrm{mmol} / \mathrm{L}$ while that in non-survivors ranged from 1.64 to $6.14 \mathrm{mmol} / \mathrm{l}$. The majority of the non-survivors had a higher lactate value of $>2 \mathrm{mmol} / \mathrm{L}$. A significant change in patient numbers was observed at different lactate levels at different time intervals ( $p$-value $<0.05$ ). Specifically, the number of patients with lactate value $>4 \mathrm{mmol} / \mathrm{L}$ changed from 4 to 18 in 72 hours, as shown in Table 2 . 


\section{Cureus}

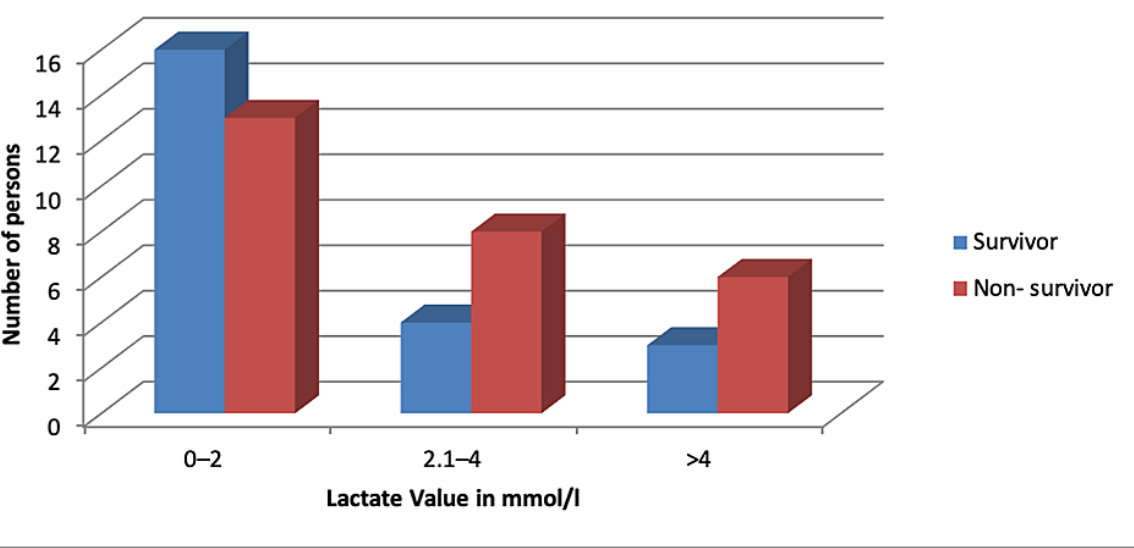

FIGURE 2: Distribution of study participants with different lactate values

Lactate Value $(\mathrm{mmol} / \mathrm{l})$
$0-2$
$2.1-4$
$>4$

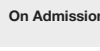

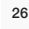

20

26

\section{At 24 Hours}

22

18

10

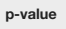

20

12

0.016

TABLE 2: Distribution of study participants with different lactate values over time

\section{Acid-base variables between the two groups}

The changes in different acid-base variables were studied over time to standardize the impact on patient outcomes, as shown in Table 3. The t-test to test the mean difference revealed significant differences in mean bi-carbonate levels at admission, 24 hours, and 72 hours between survivors and non-survivors ( $\mathrm{p}$ value $<0.01$ ). In the case of lactate levels, it was observed that non-survivors had elevated mean lactate of $>2$ $\mathrm{mmol} / \mathrm{l}$ at different time intervals, and compared to those among survivors, the difference in the lactate levels of non-survivors at different time intervals was statistically significant ( $p$-value $<0.05$ ). It is also observed that the $\mathrm{pH}$ of non-survivors was lower than survivors, and the mean $\mathrm{pH}$ value was significantly different at different time intervals among the two groups ( $\mathrm{p}$-value $<0.05$ ). Reduced $\mathrm{pH}$ levels and increased lactate levels over time, thus indicate a rise in metabolic acidosis among patients resulting in a grave outcome. 


\section{Cureus}

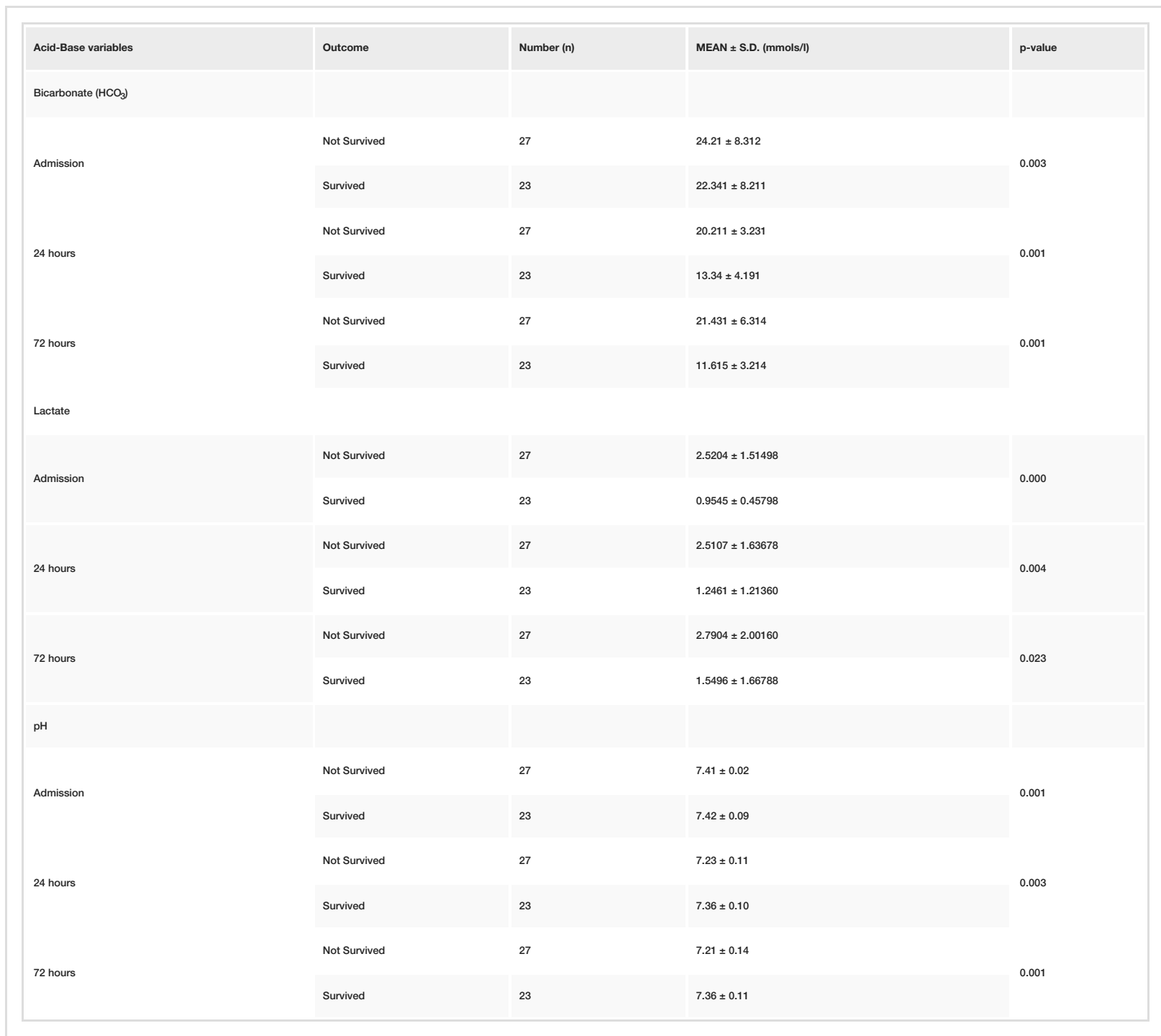

TABLE 3: Changes in mean values of acid-base variables over time

\section{Discussion}

The scientific evaluation in 50 adult critically ill patients hospitalized in CCU had a reasonable mortality precision. Like a recent study, we found that the fatal group was of a substantially higher age [10], and the death is significantly $(\mathrm{p}<0.001)$ higher within the first 24 hours in the elders [11-12], as mentioned in some reviews.

In line with some previous studies, we found a gradual increase in temperature, low mean arterial pressure [13], an increasing trend in mean heart rate [14] and mean respiratory rate [15], lower SpO2, and a significant decrease in urinary output [16] was independently associated with the non-survivors. The above studies have reported similar clinical features among the non-survivors and survivors in a different time interval on admission, 24 hours, and 72 hours in-hospital stay. The significant difference found in oxygen saturation between the survivors and non-survivors groups of the present research was in agreement with a review [16].

The association of raised blood lactate with the state of tissue hypoxia is mentioned in a review in patients with overt circulatory failure. The higher lactate values in sepsis in non-survivors were consistent with some studies $[11,15,17]$. Another study revealed similar blood lactate levels in the non-survivors initially $(3.1 \pm 2.3$ $\mathrm{mmol} / \mathrm{l}$ in non-survivors and $2.2 \pm 1.0 \mathrm{mmol} / \mathrm{l}$ in survivors) but had higher lactate levels after 12 hours ( $2.9 \pm 1.7 \mathrm{mmol} / \mathrm{l}$ in non-survivors and $1.6 \pm 0.9 \mathrm{mmol} / \mathrm{l}$ in survivors), after 24 hours $(2.1 \pm 0.6 \mathrm{mmol} / \mathrm{l}$ in nonsurvivors and $1.5 \pm 0.7 \mathrm{mmol} / \mathrm{l}$, in survivors), and after 48 hours $(2.7 \pm 1.8 \mathrm{mmol} / \mathrm{l}$ in non-survivors and $1.9 \pm 1.4$ $\mathrm{mmol} / \mathrm{l}$, in survivors) as compared with the survivors [18]. All these findings were in agreement with the present study. 
Lactate clearance had a significant inverse relationship with mortality, as the decrease in blood lactate levels in the first 24 hours significantly give a good prognosis [13]. This study concurs with our research results, where we noticed that the mean arterial blood lactate range among non-survivors was increasing at the given intervals of time [19]. The high lactate level relating to high mortality found in the current study agrees with some reviews $[16,20]$.

Like a previous review, we found decreased arterial bicarbonate concentration in the survivors' group after 24 and 72 hours [18]. Similar findings of arterial bicarbonate concentration in the non-survivors groups were noted, which increased after 72 hours as compared to 24 hours. The current findings were comparable with a review [13], where the $\mathrm{pH}$ results were $7.40 \pm 0.07$ in survivors and $7.37 \pm 0.09$ in non-survivors and the $\mathrm{p}$-value was $\mathrm{p}<0.01$.

Severity scores are essential assistants in managing sepsis patients in the CCC to predict the patient's outcome. It also helps in clinical decisions to find out a low-cost hospital stay. Some of the severity scales established for sepsis patients in the CCC during the last few decades are acute physiology and chronic health evaluation II (APACHE II), simplified acute physiology score (SAPS II), multiple organ dysfunction score (MODS), sequential organ failure assessment (SOFA), logistic organ dysfunction score (LODS), mortality prediction model II (MPM II) on admission, 24 hours, 48 hours, 72 hours, organ dysfunction and infection system (ODIN), three-day recalibrating ICU outcomes (TRIOS), and Glasgow coma score (GCS) [21].

APACHE II is the severity of the disease grading system developed in 1985 using the North American ICU patients' database [22]. The score is based on the physiologic measurement of the variable. The current study has used physiologic markers as mortality predictors, viz., temperature, blood pressure, heart rate, the rate of respiration, arterial $\mathrm{pH}$, and $\mathrm{SpO} 2$ as mentioned in the above guideline. The SAPS II system was first described in 1992 to score the severity of the patient admitted to ICU. This system also includes physiologic variables besides age, admission type, and three-disease-related variables [23], and the current study partially used some of its variables. The MODS, an objective scale, has come up in 1995 to determine the severity of multiple organ dysfunction in sepsis patients [24]. However, the current study has not used this scoring system based on six organ failure scores. In 1994, the SOFA system was used by the European Society of Intensive Care Medicine, which was revised again in 1996. This qualified system of the severity of sepsis patient based on the degree of six organ malfunctions [25], which was not used in the current study. The LODS system was proposed primarily by Le Gall et al. in 1996 using 12 six organ failures [26]. The present study has not used this model to score predictive of the patient's outcome at ICU. MPM II, primarily described by Lemeshow et al. [27], evaluated the hospital death directly following sepsis at 24, 48, and 72 hours like that of the current study. The ODIN system was initiated by Fagon et al. This scoring system uses the recorded data within the first 24 hours at ICU admission to observe any malfunction of six organs, one infection, and the differentiates of prognosis [28]. In 2001, Timsit et al. [29] projected the TRIOS with daily SAPS II and LODS for sepsis patients admitted in the ICU for mortality prediction at 72 hours. The GCS is a worldwide tool for the fast calculation of an injured patient's consciousness level [30]. There was no sepsis case following injury; hence, we did not follow this mortality predictor.

Too many tasks determine the sepsis cases' hospital outcome for real-time decision-making for an effective and low-cost hospital stay. The clinical and biochemical predictors evaluated in the current study are convenient markers to determine in a set-up like ours, outreaching the updated technology to find out a low-cost management strategy.

\section{Limitations of the study}

The small sample size would have resulted in a less precise estimation of the frequency of different variables we studied. Besides, this study was conducted in a single-center, so the results may not be generalized to other centers explicitly dedicated to the management of sepsis patients. Also, the patients were followed up for four weeks. Hence, a more extensive multicentre study with a more extended period of patient follow-up may be useful.

\section{Conclusions}

Changes in the vital clinical signs of patients suffering from sepsis were found at different time intervals during examination and management. Furthermore, the changes in acid-base variables during the course of admission could be useful determinants in predicting patient morbidity and mortality. Also, serial lactate levels, bi-carbonate levels, and pH levels may be significant clinical predictors of patient mortality.

\section{Additional Information \\ Disclosures}

Human subjects: Consent was obtained or waived by all participants in this study. Ethics Committee of Assam Medical College and Hospital issued approval AMC/EC/PG/100. Animal subjects: All authors have confirmed that this study did not involve animal subjects or tissue. Conflicts of interest: In compliance with the ICMJE uniform disclosure form, all authors declare the following: Payment/services info: All authors have declared that no financial support was received from any organization for the submitted work. 
Financial relationships: All authors have declared that they have no financial relationships at present or within the previous three years with any organizations that might have an interest in the submitted work. Other relationships: All authors have declared that there are no other relationships or activities that could appear to have influenced the submitted work.

\section{References}

1. Peake SL, Delaney A, Bailey M, et al.: Goal-directed resuscitation for patients with early septic shock . N Engl J Med. 2014, 371:1496-506. 10.1056/NEJMoa1404380

2. Mouncey PR, Osborn TM, Power GS, et al.: Trial of early, goal-directed resuscitation for septic shock . N Engl J Med. 2015, 372:1301-1311. 10.1056/NEJMoa1500896

3. Dellinger RP, Levy MM, Rhodes A, et al.: Surviving sepsis campaign. International guidelines for management of severe sepsis and septic shock. Crit Care Med. 2012, 41:580-637. 10.1097/CCM.0b013e31827e83af

4. Shankar-Hari M, Phillips GS, Levy ML, et al.: Developing a new definition and assessing new clinical criteria for septic shock. For the third international consensus definition for sepsis and septic shock (sepsis-3). JAMA. 2016, 315:755-787. 10.1001/JAMA.2016.0289

5. Bakker J, Nijsten NM, Jansem CT: Clinical use of lactate monitoring in critically ill patients . Annals of Intensive Care. 2013, 3:12. https://doi.org/10.1186/2110-5820-3-12

6. Jansen TC, Van BJ, Bakker J: Blood lactate monitoring in critically ill patients: a systematic health technology assessment. Crit Care Med. 2009, 37:2827-2839. 10.1097/ccm.0b013e3181a98899

7. Shapiro NI, Howell MD, Talmor D, Nathanson LA, Lisbon A, Wolfe RE, Weiss JW: Serum lactate as a predictor of mortality in emergency department patients with infection. Ann Emerg Med. 2005, 45:524-528. 10.1016/j.annemergmed.2004.12.006

8. Levraut J, Ciebiera JP, Chave S, Rabary O, Jambou P, Carles M, Grimaud D: Mild hyperlactatemia in stable septic patients is due to impaired lactate clearance rather than overproduction. Am J Respir Crit Care Med. 1998, 157:1021-1026. 10.1164/ajrccm.157.4.9705037

9. Rhodes A, Laura EE, Alhazzani W, et al.: Surviving Sepsis Campaign: International guidelines for management of sepsis and septic shock: 2018. Intensive Care Med. 2017, 43:304-377. 10.1007/s00134-0174683-6

10. Bart H, Eck RJ, Renske W, et al.: Clinical examination for the prediction of mortality in the critically ill: the simple intensive care studies-I. Crit Care Med. 2019, 47:1301-1309. 10.1097/CCM.0000000000003897

11. Blum A, Almajid Zoubi A, Kuria S, Blum N: High serum lactate level may predict death within 24 hours . Open Med (Wars). 2015, 10:318-322. 10.1515/med-2015-0045

12. Duke TD, Butt W, South M: Predictors of mortality and multiple organ failure in children with sepsis . Intensive Cre Med. 1997, 23:684-692. 10.1007/s001340050394

13. Bakker J, Gris P, Coffernils M, Kahn RJ, Vincent J-L: Serial blood lactate levels can predict the development of multiple organ failure following septic shock. Am J Surg. 1996, 171:221-226. 10.1016/S00029610(97)89552-9

14. Lavery RF, Livingston DH, Tortella BJ, Sambol JT, Slomovitz BM, Siegel JH: The utility of venous lactate to triage injured patients in the trauma center. J Am Coll Surg. 2000, 190:656-664. 10.1016/s10727515(00)00271-4

15. Krishna U, Joshi PS, Modh M: An evaluation of serial blood lactate measurement as an early predictor of shock and its outcome in patients of trauma or sepsis. Indian J Crit Care Med. 2009, 13:66-73. 10.4103/09725229.56051

16. Bou R, Christopher EK, Shamil A, Rajha E, Faris N, Bachir R, Dagher GA: Serum lactate is an independent predictor of hospital mortality in critically ill patients in the emergency department: a retrospective study. Scand J Trauma Resusc Emerg Med. 2017, 25:69. 10.1186/s13049-017-0415-8

17. Sanderson M, Chikhani M, Blyth E, Wood S, Moppett IK, McKeever T, Simmonds MJR: Predicting 30-day in patients with sepsis: an exploratory analysis of process of care and patient characteristics. J Intensive Care Soc. 2018, 19:299-304. 10.1177/1751143718758975

18. Megrelli A, Oliveria PR, Friedman G: Occult hypoperfusion is associated with increased mortality in hemodynamically stable, high-risk, surgical patients. Crit Care. 2004, 8:R60-R65. 10.1186/cc2423

19. Nguyen HB, Rivers EP, Knoblich BP, Jacobsen G, Muzzin A, Ressler JA, Tomlanovich MC: Early lactate clearance is associated with improved outcome in severe sepsis and septic shock. Crit Care Med. 2004, 32:1637-1642. 10.1097/01.ccm.0000132904.35713.a7

20. Yong JP, Dong HK, Seong CK, et al.: Serum lactate upon emergency department arrival as a predictor of 30day in-hospital mortality in an unselected population. PloS One. 2018, 13:e0190519. 10.1371/journal.pone.0190519

21. Rapsang AG, Shyam DC: Scoring systems in the intensive care unit: a compendium . Indian J Crit Care Med. 2014, 18:220-228. 10.4103/0972-5229.130573

22. Knaus WA, Draper EA, Wagner DP, Zimmerman JE: APACHE II: a severity of disease classification system . Crit Care Med. 1985, 13:818-829.

23. Le Gall JR, Lemeshow S, Saulnier F: A new simplified acute physiology score (SAPS II) based on a European/North American multicenter study. JAMA. 1993, 270:2957-2963. 10.1001/jama.270.24.2957

24. Marshall JC, Cook DJ, Christou NV, Bernard GR, Sprung CL, Sibbald WJ: Multiple organ dysfunction score: a reliable descriptor of a complex clinical outcome. Crit Care Med. 1995, 23:1638-1652. 10.1097/00003246199510000-00007

25. Vincent JL, Moreno R, Takala J, et al.: The SOFA (Sepsis-related Organ Failure Assessment) score to describe organ dysfunction/failure. Intensive Care Med. 1996, 22:707-710. 10.1007/BF01709751

26. Le Gall JR, Klar J, Lemeshow S, Saulnier F, Alberti C, Artigas A, Teres D: The logistic organ dysfunction system. A new way to assess organ dysfunction in the intensive care unit. ICU Scoring Group. JAMA. 1996, 276:802-810.

27. Lemeshow S, Klar J, Teres D, Avrunin JS, Gehlbach SH, Rapoport J, Rué M: Mortality probability models for 


\section{Cureus}

patients in the intensive care unit for 48 or 72 hours: A prospective, multicenter study. Crit Care Med. 1994, 22:1351-1358. 10.1097/00003246-199409000-00003

28. Fagon JY, Chastre J, Novara A, Medioni P, Gibert C: Characterization of intensive care unit patients using a model based on the presence or absence of organ dysfunctions and/or infection: the ODIN model. Intensive Care Med. 1993, 19:137-144. 10.1007/BF01720528

29. Timsit JF, Fosse JP, Troché G, et al.: Accuracy of a composite score using daily SAPS II and LOD scores for predicting hospital mortality in ICU patients hospitalized for more than $72 \mathrm{~h}$. Intensive Care Med. 2001, 27:1012-1021. 10.1007/s001340100961

30. Teasdale G, Jennett B: Assessment of coma and impaired consciousness. A practical scale . Lancet. 1974, 2:81-84. 10.1016/s0140-6736(74)91639-0 